\title{
Design, synthesis, and characterization of novel nanowire structures for photovoltaics and intracellular probes
}

\section{Citation}

Tian, Bozhi, and Charles M. Lieber. 2011. “Design, Synthesis, and Characterization of Novel Nanowire Structures for Photovoltaics and Intracellular Probes." Pure and Applied Chemistry 83 (12) (January 31). doi:10.1351/pac-con-11-08-25.

\section{Published Version}

10.1351/pac-con-11-08-25

\section{Permanent link}

http://nrs.harvard.edu/urn-3:HUL.InstRepos:34520377

\section{Terms of Use}

This article was downloaded from Harvard University's DASH repository, and is made available under the terms and conditions applicable to Other Posted Material, as set forth at http:// nrs.harvard.edu/urn-3:HUL.InstRepos:dash.current.terms-of-use\#LAA

\section{Share Your Story}

The Harvard community has made this article openly available.

Please share how this access benefits you. Submit a story.

\section{Accessibility}


Pure Appl. Chem., Vol. 83, No. 12, pp. 2153-2169, 2011.

doi:10.1351/PAC-CON-11-08-25

(C) 2011 IUPAC, Publication date (Web): 31 October 2011

\title{
Design, synthesis, and characterization of novel nanowire structures for photovoltaics and intracellular probes *
}

\author{
Bozhi Tian ${ }^{1, \ddagger}$ and Charles M. Lieber ${ }^{2}$ \\ ${ }^{1}$ David H. Koch Institute for Integrative Cancer Research, Massachusetts Institute \\ of Technology, Cambridge, MA 02139, USA; ${ }^{2}$ Department of Chemistry and \\ Chemical Biology, School of Engineering and Applied Sciences, Harvard \\ University, Cambridge, MA 02138, USA
}

\begin{abstract}
Semiconductor nanowires (NWs) represent a unique system for exploring phenomena at the nanoscale and are expected to play a critical role in future electronic, optoelectronic, and miniaturized biomedical devices. Modulation of the composition and geometry of nanostructures during growth could encode information or function, and realize novel applications beyond the conventional lithographical limits. This review focuses on the fundamental science aspects of the bottom-up paradigm, which are synthesis and physical property characterization of semiconductor NWs and NW heterostructures, as well as proofof-concept device concept demonstrations, including solar energy conversion and intracellular probes. A new NW materials synthesis is discussed and, in particular, a new "nanotectonic" approach is introduced that provides iterative control over the NW nucleation and growth for constructing 2D kinked NW superstructures. The use of radial and axial $p$-type/intrinsic/n-type $(p-i-n)$ silicon NW (Si-NW) building blocks for solar cells and nanoscale power source applications is then discussed. The critical benefits of such structures and recent results are described and critically analyzed, together with some of the diverse challenges and opportunities in the near future. Finally, results are presented on several new directions, which have recently been exploited in interfacing biological systems with NW devices.
\end{abstract}

Keywords: biotechnology; intracellular; nanowires; photovoltaics; synthesis.

\section{INTRODUCTION}

Semiconductor nanowires (NWs) [1-8], nanocrystals [9-11], and carbon nanotubes [12-16] offer many opportunities for the assembly of nanoscale devices and arrays by the bottom-up paradigm [1-4]. Moreover, these nanomaterials demonstrate new and/or enhanced functions crucial to many areas of technology. Central to realizing applications through a bottom-up paradigm is the rational control of key nanomaterial parameters, including chemical composition, structure, size, morphology, and doping. It is these parameters that determine, for example, electronic and optoelectronic properties critical to predictable device function. Significantly, semiconductor NWs represent the nanomaterial system where these key parameters have been best controlled to date, have become a unique system for exploring phe-

\footnotetext{
*Pure Appl. Chem. 83, 2115-2212 (2011). A collection of invited, peer-reviewed articles by the winners of the 2011 IUPAC Prize for Young Chemists.

¥Corresponding author
} 
nomena at the nanoscale, and are also expected to play a critical role in future electronic and optoelectronic devices.

In this review, we first introduce a new "nanotectonic" approach that provides iterative control over the NW nucleation and growth for constructing 2D kinked NW superstructures [17]. Next, a rational, multistep approach toward the general synthesis of 3D branched NW heterostructures [18] is discussed, together with results on novel nanoscale electronic devices based on those new NW structures, e.g., self-labeled $p-n$ diodes and field-effect transistors (FETs) in kinked NWs [17] and addressable light-emitting diode (LED) array and biological sensors in branched NWs [18].

Efforts toward using radial and axial $p$-type/intrinsic/n-type $(p-i-n)$ silicon NW ( $\mathrm{Si}$-NW) building blocks for solar cells and nanoscale power source applications [19-22] include discussion of the critical benefits of such structures, description of recent results, and critical analysis and insights into diverse challenges and opportunities in the near future.

Finally, we present results on two new directions that have recently been exploited in interfacing biological systems with NW devices. It is first shown that Si-NW FET arrays fabricated on transparent substrates can be reliably interfaced to acute brain slices, and can be used to reveal spatially heterogeneous functional connectivity in the olfactory cortex with high spatio-temporal resolutions [23]. Next, we demonstrate the first electrical recording of the intracellular potential with 3D nanoprobe devices [24]. Significantly, electrical recordings of spontaneously beating cardiomyocytes demonstrate that our 3D NW probes can continuously monitor the extra- to intracellular signals during cellular uptake [24]. The nanometer size, biomimetic surface coating, and flexible 3D device geometry make these active semiconductor nanoprobes new and powerful tools for intracellular measurements, and suggest future biomedical applications where the distinctions between living cells and electronic devices have been blurred [24].

\section{NEW NANOWIRE MATERIALS}

\section{Nanowire synthesis overview}

In general, synthesis of NWs proceeds via nanocluster-catalyzed vapor-liquid-solid (VLS) growth [25]. In this process, the metal nanoclusters are heated above the eutectic temperature for the metal-semiconductor system of choice in the presence of a vapor-phase source of the semiconductor, resulting in a liquid droplet of the metal-semiconductor alloy. The continued feeding of the semiconductor reactant into the liquid droplet supersaturates the eutectic, leading to nucleation of the solid semiconductor. The solid-liquid interface forms the growth interface, which acts as a sink, causing the continued semiconductor incorporation into the lattice and, thereby, the growth of the NW with the alloy droplet riding on the top.

The gaseous semiconductor reactants can be generated through decomposition of precursors in a chemical vapor deposition (CVD) process, or through momentum and energy-transfer methods such as pulsed laser ablation [26] or molecular beam epitaxy (MBE) [27] from solid targets. So far, CVD has been the most popular technique. In CVD-VLS growth, the metal nanocluster serves as a catalyst at which site the gaseous precursors decompose, providing the gaseous semiconductor reactants. In the case of $\mathrm{Si}-\mathrm{NW}$ growth, silane $\left(\mathrm{SiH}_{4}\right)$ and $\mathrm{Au}$ nanoparticles (Au-NPs) are normally used as the precursor and catalysts, respectively. Besides group IV materials, compound III-V and II-VI NWs have also been produced with the VLS method, in which pseudo-binary phase diagrams for the catalyst and compound semiconductor of interest are employed. In the compound semiconductor case, metal-organic chemical vapor deposition (MOCVD) [28] or pulsed laser ablation [29] are typically used to provide the reactants. 


\section{Kinked nanowires}

The ability to control and modulate the composition [30-32], doping [30,32,33], crystal structure [34-36], and morphology [37,38] of semiconductor NWs during the synthesis process has allowed researchers to explore various applications of NWs [39,40]. Despite these advances in NW synthesis [30-38], progress toward the ab initio design and growth of hierarchical nanostructures has been limited. In order to encode distinct structural units within a single NW, we have developed a novel approach that provides iterative control over the nucleation and growth of NWs [17]. In our experiments, we introduced kinks at defined positions during growth, and all the kinked units are confined to a single plane. These hierarchical NWs are built up using a "nanotectonic" approach analogous to metal-organic framework materials [41], where we define a "secondary building unit" (SBU) consisting of two straight single-crystalline arms (blue, Fig. 1A) connected by one fixed $120^{\circ}$ angle joint (green, Fig. 1A).

A
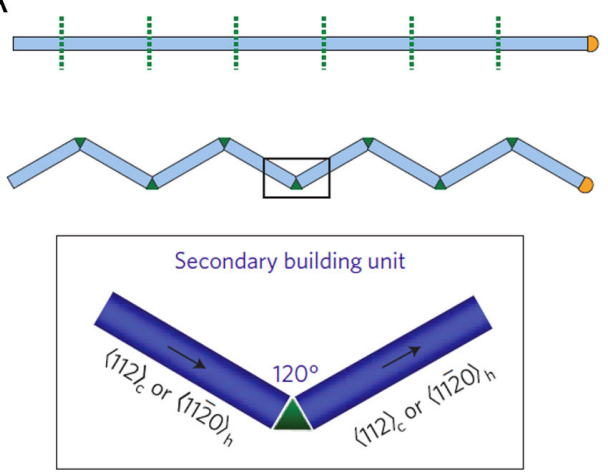

C
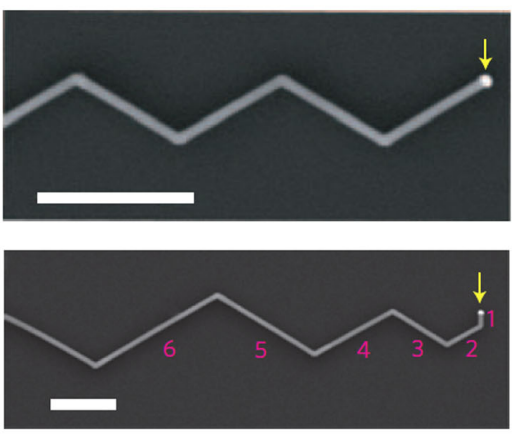

$\mathrm{B}$

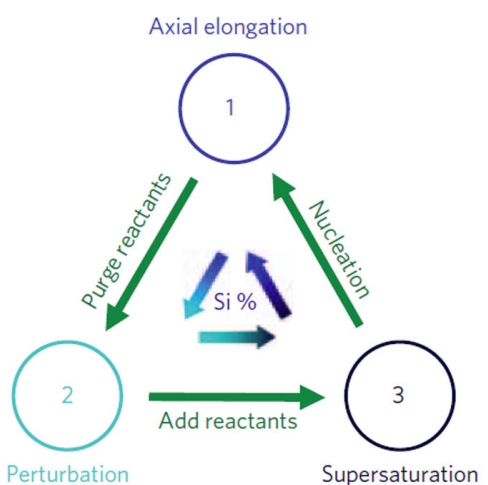

$\mathrm{D}$

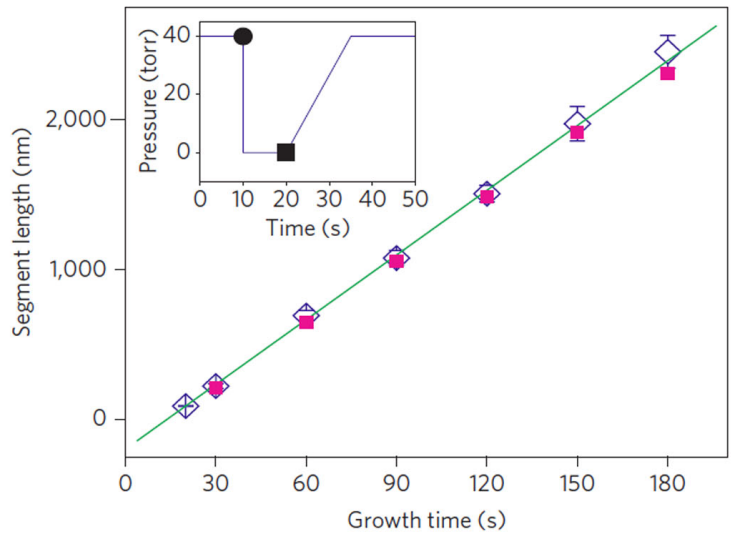

Fig. 1 Design and controlled synthesis of multiply kinked NWs. A, Schematic of a coherently kinked NW and the SBU, which contains two arms (blue) and one joint (green). Subscripts $\mathrm{c}$ and $\mathrm{h}$ denote cubic and hexagonal structures, respectively. B, Cycle for the introduction of an SBU by stepwise synthesis. The color gradient accompanying the innermost blue arrows indicates the change of Si concentration in nanocluster catalyst during synthesis of a kinked Si-NW. C, SEM image of a multiply kinked 2D Si-NW with equal (upper panel) and decreasing (lower panel) arm segment lengths. Scale bar, $1 \mu \mathrm{m}$. The yellow arrow highlights the position of the nanocluster catalyst. D, Plot of segment length vs. growth time. Each blue diamond represents average segment length data (error bars: \pm 1 s.d.) from a sample containing NWs with uniform segment lengths between kinks. The green line is a linear fit to these data. Magenta solid squares are data points taken from the NW shown in $\mathbf{C}$ (lower panel). Inset, growth pressure variation during kink synthesis. The black solid sphere and square denote the start of purging and re-introduction of reactants, respectively. 
We note that two $\langle 112\rangle_{\mathrm{c}}$ or $\langle 110\rangle_{\mathrm{c}}$ vectors in a cubic crystal structure, and two $\langle 11-20\rangle_{\mathrm{h}}$ or $\langle 1-100\rangle_{\mathrm{h}}$ vectors in a hexagonal structure can form the desired $120^{\circ}$ joint when rotating about the $\langle 111\rangle_{c}$ and $<0001\rangle_{\mathrm{h}}$ zone axes, respectively. SBU formation involves three main steps during nanocluster-catalyzed growth (Fig. 1B); (1) axial growth of a 1D NW arm segment, (2) purging of gaseous reactants to suspend NW elongation, and (3) supersaturation and nucleation of NW growth following re-introduction of reactants. Steps 1-3 can be iterated to link a number of SBUs generating a 2D chain structure.

The synthetic approach is general and can be applied to the growth of kinked group IV, II-VI, and III-V semiconductor NWs. Moreover, while the length and number of straight segments between the triangular joints can be controlled, the crystallographic growth direction is preserved throughout the synthesis.

Our results highlight an emerging potential of our bottom-up "nanotectonic" approach to generate more complex NWs with potentially unique function "integrated" at the nanoscale in the topologically defined points of the kinks [17]. We illustrate this capability by combining our iterative growth approach with additional modulation of dopant to vary electronic characteristics in a well-defined manner with respect to the kinks. A kinked Si-NW SBU with integrated $n$ - and $p$-type arms was synthesized by switching phosphine and diborane dopants during the kink growth sequence. Current-voltage $(I-V)$ data recorded on a representative single kink device (Fig. 2A) reveal a clear current rectification in reverse bias with an onset at forward bias voltage of $\sim 0.6 \mathrm{~V}$, consistent with the synthesis of a welldefined $p$ - $n$ diode within the kinked structure. Moreover, an electrostatic force microscopy image of a typical kinked $p-n \mathrm{NW}$ in reverse bias (Fig. 2B) showed that the voltage drop occurs primarily at the designed $p-n$ junction localized and labeled by the kink during growth.

In addition, our concept can be extended to design and synthesis of NWs with distinct functionality at sequential kinks [17]. A representative atomic force microscopy image of a double kink structure synthesized with $n^{+}$and $n$ dopant profiles at the two kink joints (Fig. 2C) shows that the characteristic SBU described above is unaffected by multiple modulations of dopant concentration. Notably, scanned gate microscopy data (Fig. 2D) demonstrate enhanced (decreased) NW conductance as the tip with positive (negative) gate potential is scanned across the designed $n$-type segment immediately adjacent to the upper-left kink junction, thus confirming the integration of an $n$-type FET at a well-defined and recognizable point on the structure. The absence of gate response from the lower-right kink junction (Fig. 2D) further shows that the single-crystalline kink structure itself will not alter the electrical transport properties. We believe that these synthetic results and demonstrated topologically defined functional devices represent a significant advance toward the realization of ab initio designed and "selflabeled" 2D NW structures. Such designed and self-labeled 2D NW structures may open up unique applications in bottom-up integration of active devices in nanoelectronics, photodetector arrays, multiplexed biological sensors, and the presentation of multiterminal nanodevices in 3D. 

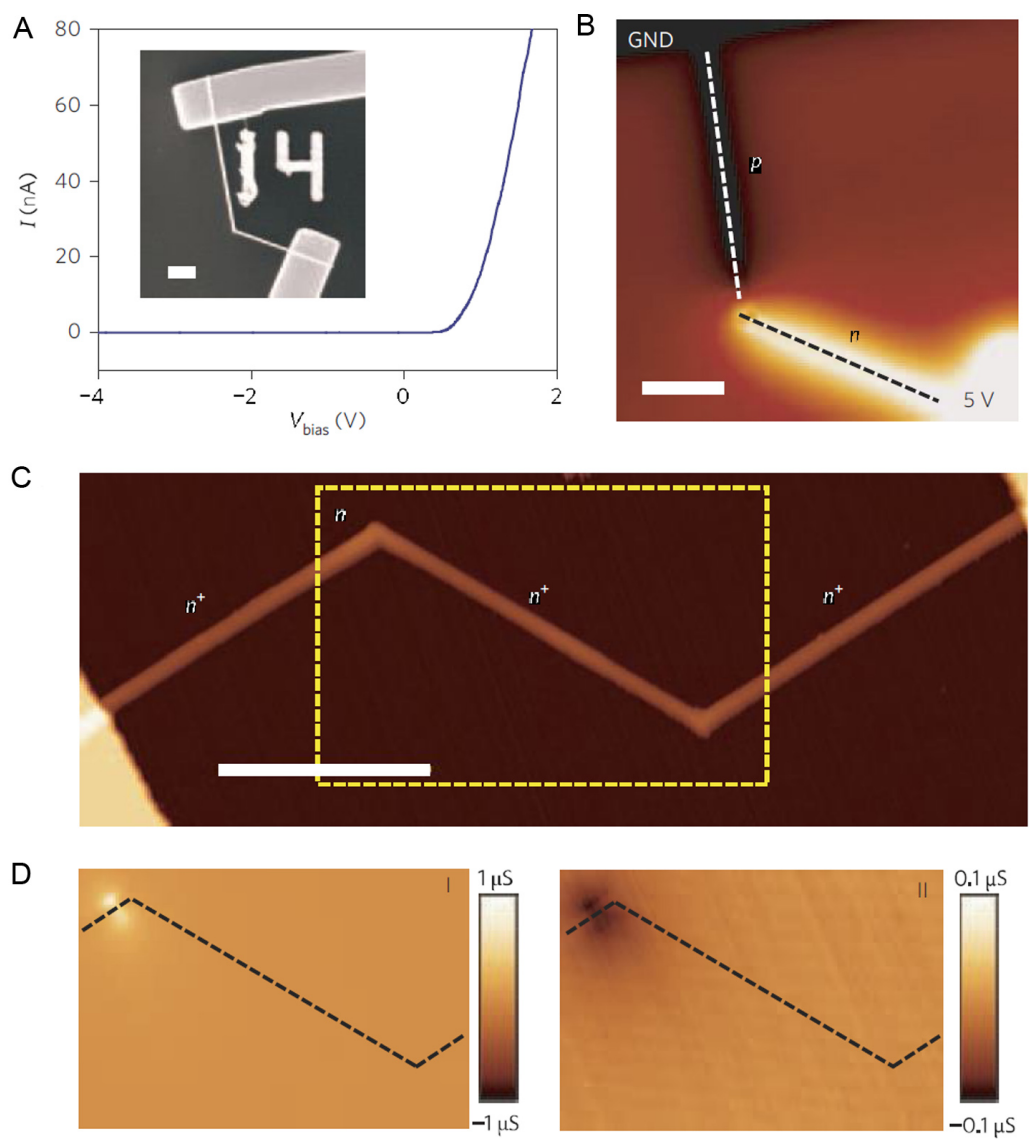

Fig. 2 Topologically defined nanoelectronic devices. A, $I-V$ data recorded from a kinked $p-n$ Si-W device. Inset, SEM image of the device structure; scale bar is $2 \mu \mathrm{m}$. B. Electrostatic force microscopy image of a $p$ - $n$ diode reverse-biased at $5 \mathrm{~V}$. The AFM tip voltage was modulated by $3 \mathrm{~V}$ at the cantilever-tip resonance frequency. The signal brightness is proportional to the NW device surface potential, and shows an abrupt drop around the kink position. The dashed lines mark the NW position. Scale bar is $2 \mu \mathrm{m}$. $\mathbf{C}$ and $\mathbf{D}, \mathrm{AFM}$ and scanning gate microscopy images of one $n^{+}-k i n k-n^{+}-k i n k-\left(n-n^{+}\right)$dopant-modulated double-kinked Si-NW structure. The scale bar in $\mathbf{c}$ is $2 \mu \mathrm{m}$. The scanning gate images were recorded with a $V_{\text {tip }}$ of $10 \mathrm{~V}$ (I) and $-10 \mathrm{~V}$ (II), respectively, and $V_{\text {sd }}$ of $1 \mathrm{~V}$. The dark and bright regions correspond to reduced and enhanced conductance, respectively. The black dashed lines mark the NW position.

\section{Heterobranched nanowires}

In order to extend further our topographical control over NW growth, we have demonstrated a rational, multistep approach to the synthesis of branched NW heterostructures [18]. We have focused on two distinct classes of branched NWs, with metal or semiconductor branches grown on either the native surface of semiconductor (type I, Fig. 3) or on the oxide surface of core/shell semiconductor/oxide (type II) NW backbones. The synthesis involves two critical steps following synthesis of the core and core/shell NWs. First, Au-NPs are selectively deposited onto the respective backbone surfaces using either an in situ solution reduction of $\mathrm{AuCl}^{4-}$ on Si-NW surfaces for type I structures (Fig. 3) or binding of $\mathrm{Au}-\mathrm{NPs}$ to the oxide surfaces of $\mathrm{Si} / \mathrm{SiO}_{2}$ core/shell NWs for type II structures. Next, branches with various compositions are grafted onto the backbone NWs through a seed-mediated growth mechanism under either gas- or solution-phase conditions. 

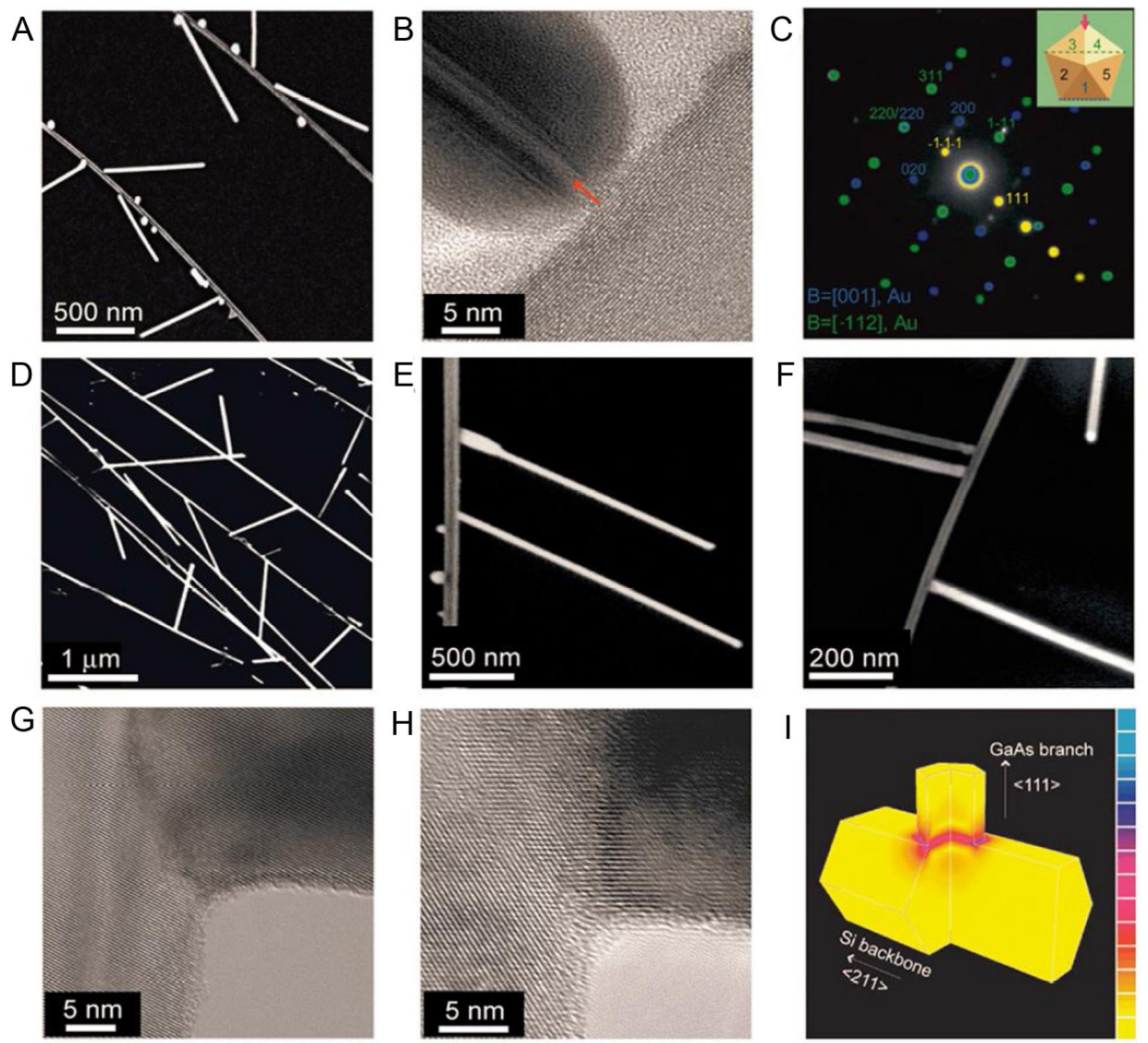

Fig. 3 Structural characterization of type I branched NW heterostructures. A, SEM image of Si/Au-branched NWs. B, High-resolution transmission electron microscopy (HRTEM) image of Si/Au branched junction; red arrow highlights twin plane. C, Selected-area electron diffraction (SAED) pattern of the junction region shown in (B), where blue and green spots originate from $\langle 100\rangle_{A u},\langle 112\rangle_{A u}$ zone diffraction, and yellow spots are from the crystalline Si backbone. (inset) Cross-sectional model of the penta-twinned Au branch consisting of five twinned subunits. Red arrow marks the incident beam direction. D-F, SEM images of Si/Ge (D), Si/GaAs (E), and Si/GaP (F) branched NWs. G and H, HRTEM images of $\mathrm{Si} / \mathrm{Ge}(\mathbf{G})$ and $\mathrm{Si} / \mathrm{GaAs}(\mathbf{H})$ branched junctions. I, Simulated von Mises stress field at $\mathrm{Si} / \mathrm{GaAs}$ branched junction. The scale bar range is from $3.1 \times 10^{6}$ to $1.6 \times 10^{10} \mathrm{~Pa}$.

Single-crystalline semiconductor (IV-IV, III-V, II-VI) and metal branches have been grown on either bare (type I) or core/shell (type II) NW backbones with explicit control over composition, morphology, and doping (Fig. 3). Measurements made on the different composition branched NW structures demonstrate encoding of functional $p$ - $n$-type diodes (type I) and LEDs (type I) as well as FETs (type II) with device function localized at the branch/backbone NW junctions. In addition, multibranch/backbone NW structures were synthesized and used to demonstrate capability to create addressable nanoscale LED arrays (type I), logic circuits (type II), and biological sensors (type II). Our work demonstrates a previously undescribed level of structural and functional complexity in NW materials, and more generally, highlights the potential of bottom-up synthesis to yield increasingly complex functional systems in the future. 


\section{SINGLE NANOWIRE PHOTOVOLTAICS}

\section{Motivation}

Solar energy conversion is a highly attractive process for clean and renewable power $[42,43]$. For over two decades, commercial single- and multicrystalline Si solar panels have dominated the world market for photovoltaics (PV), and in recent years growth in production of solar cell units has averaged $30 \%$ per year [44]. More recent developments in commercial PV have centered on second-generation solar cells based on thinner semiconductor absorber layers such as $\mathrm{CuIn}_{x} \mathrm{Ga}_{1-x} \mathrm{Se}_{2}, \mathrm{CdTe}$, and amorphous $\mathrm{Si}$ [42-45]. In addition, organic and dye/electrolyte-based solar cells are currently in active research and development [46-48], and some are at preliminary stages of commercialization. These latter technologies provide solar energy conversion at lower cost than established Si technology, however, they do not yet achieve the efficiency of well-established multicrystalline PVs. Third-generation solar cells, including tandem cells $[45,49,50]$ and down-converter cells [45], have demonstrated energy conversion efficiencies beyond the Shockley-Quiessier limiting efficiency of $31 \%$ for a single cell at one-sun illumination, but at much higher cost. Due to their excellent material quality and tunable bandgaps, III-V compound semiconductors are normally key components of such high-efficiency solar cells. In particular, a three-junction GaInP/GaInAs/Ge cell has recently surpassed the $40 \%$ efficiency milestone [50]. Moreover, studies attempting to harness effects such as multiple exciton generation and hot carriers in quantum confined nanostructures have also created much interest recently [51]. These ideas suggest that fundamental studies of the synthesis and PV properties of nanoscale structures could serve as a rich area in which to search for high-efficiency and low-cost solar cell solutions.

As a prototypical example, semiconductor NWs are a broad class of materials which, through controlled growth and organization, have led to a number of novel nanoscale photonic and electronic devices [52]. Given the need to elucidate the intrinsic characteristics and limits of nano-enabled solar cells in order to evaluate their potential for next-generation, large-scale, high-efficiency, and low-cost solar cells [19-22,53-57], as well as integrated power solutions for emerging nanoelectronic devices, we have initiated studies exploiting single NW heterostructures as stand-alone and active PV elements [19-22].

The use of single NWs as PV elements presents several key advantages which may be leveraged to produce high-efficiency, robust, integrated nanoscale PV power sources. First, the principle of bottom-up design allows the rational control of key nanomaterial parameters, which will determine PV performance, including chemical/dopant composition and diode junction structure, size, and morphology. Importantly, this principle has been demonstrated previously in a wide variety of nanoscale structures and devices [52]. Second, single or interconnected NW PV elements could be seamlessly integrated with conventional electronics and/or future nanoscale electronics to provide energy for low-power applications. Third, studies of PV properties at the single NW level will permit determination of the intrinsic limits, areas of improvement, and potential benefits of nano-enabled PV (e.g., cavity effect and photon confinement).

\section{Materials design}

Two unique structural motifs that can yield functional PV devices at the single NW level include $p-i-n$ dopant modulation in axial $[19,21]$ and radial $[19,20]$ geometries (Fig. 4A). The pink, yellow, and blue regions denote the $p-i-n$ diode segments, respectively. In this structure, electron-hole pairs are generated throughout the device upon absorption of photons whose energies are equal to or greater than the bandgap of $\mathrm{Si}\left(E_{\mathrm{g}}=1.12 \mathrm{eV}\right.$ for single-crystal $\left.\mathrm{Si}\right)$. Carrier generation and separation are most efficient within the depletion region owing to the built-in field established across the $p-i-n$ junction $[19-22,58]$. Once swept in the direction of the electric field, the photogenerated holes (electrons) traverse through the $p$-type ( $n$-type) regions and are collected as a photocurrent by ohmic metal contacts. 
A

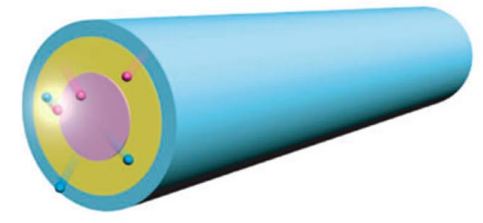

C

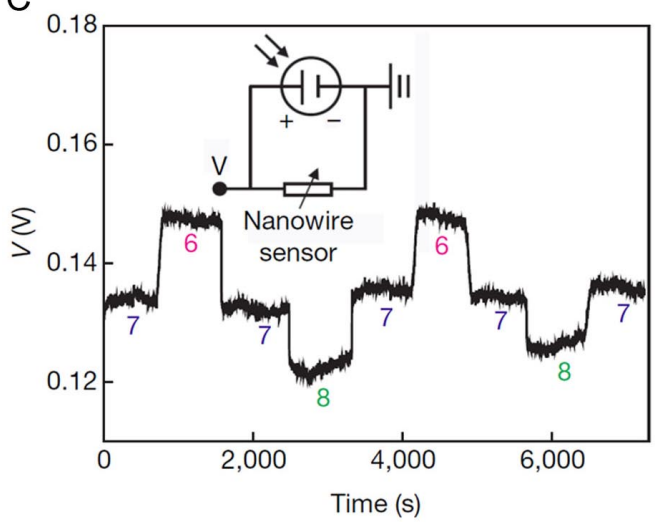

B

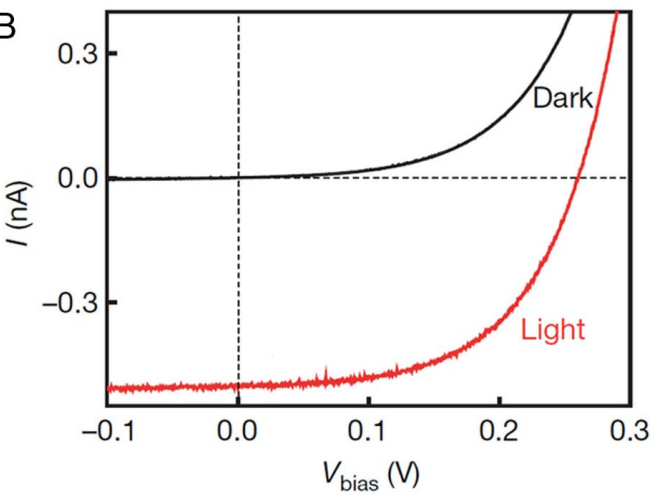

D

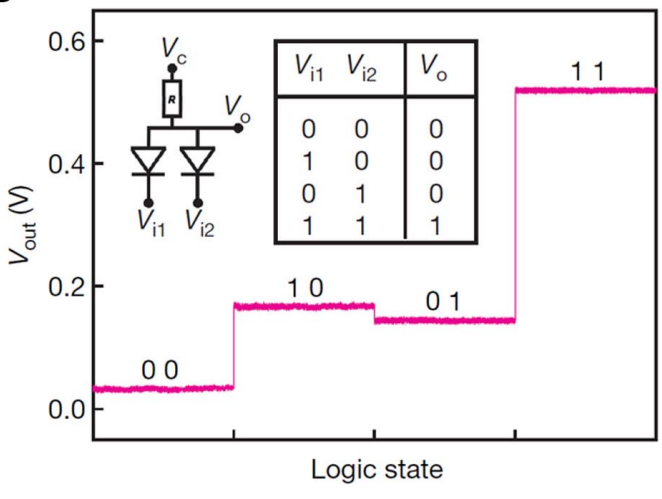

Fig. 4 Single NW photovoltaics. A, Schematic of carrier generation and separation in axial (upper) and radial (lower) $p$ - $i$ - $n$ NWs. The pink, yellow, and blue regions denote the $p$-type, $i$-, and $n$-type diode segments, respectively. The pink and blue spheres denote the holes and electrons, respectively. B, Dark and light $I-V$ curves of a coaxial Si-NW device. C, Real-time detection of the voltage drop across a modified Si-NW at different $\mathrm{pH}$ values. The Si-NW pH sensor is powered by a single Si-NW PV device. D, NW AND logic gate powered by two Si-NW PV devices in series.

In the axial configuration (Fig. 4A, upper), the $p$ - and $n$-type regions can be made arbitrarily short since their main purpose is to provide contact to the junction embedded within the NW. Therefore, the active device area can be kept very small so as to enhance integration. A closely related structure is the $p-i-n$ radially modulated NW diode (Fig. 4A, lower). The overall device physics is identical to that of the axially modulated motif, with the key added benefits being that the $p-i-n$ interface extends along the length of the NW and that carrier separation takes place in the radial vs. the longer axial direction. Since the latter yields a carrier collection distance smaller or comparable to the minority carrier diffusion length $[20,57,58]$, photogenerated carriers can reach the $p-i-n$ junction with high efficiency without substantial bulk recombination. Indeed, recent theoretical studies have suggested that coaxial NW structures could improve carrier collection and overall efficiency with respect to comparable single-crystal bulk semiconductors, and especially when relatively low quality materials are used as absorber materials [57]. Large-scale vertically aligned arrays of coaxial NWs would enable substantial light absorption along the long axis of the NWs, and also afford the benefit of short-range and efficient radial carrier separation. Together, these advantages would orthogonalize the pathways for light absorption and carrier collection [57], thereby eliminating a key limitation of conventional planar solar cells. 


\section{Device characterizations}

Under 1-sun $\left(100 \mathrm{~mW} / \mathrm{cm}^{2}\right)$ simulated solar illumination, the $p$-i-n Si-NW coaxial elements yield a maximum power output of $200 \mathrm{pW}$ per NW and an energy conversion efficiency of up to $3.4 \%$ (Fig. 4B), with stable and improved efficiencies achievable at higher intensities. Significantly, individual and interconnected Si-NW PV elements serve as robust power sources to drive functional nanoelectronic sensors and logic gates (Fig. 4C, 2D).

We also reported the first experimental realization of axial modulation-doped $p-i-n$ and tandem $p-i-n^{+}-p^{+}-i-n$ Si-NW PV elements. Under 1-sun simulated solar conditions, optimized $p-i-n$ Si-NW devices exhibited an open-circuit voltage $\left(V_{\mathrm{oc}}\right)$ of $0.29 \mathrm{~V}$, a maximum short-circuit current density of $3.5 \mathrm{~mA} / \mathrm{cm}^{2}$, and a maximum efficiency of $0.5 \%$. In addition, a novel single Si-NW tandem solar cell consisting of the synthetic integration of two PV elements with an overall $p-i-n^{+}-p^{+}-i-n$ structure was prepared and shown to exhibit a $V_{\text {oc }}$ that is on average $57 \%$ larger than that of the single $p-i-n$ device. This result underscores the potential for facile integration of multiple PV cells at the nanoscale.

The aforementioned coaxial and axial Si-NW PV elements provide a new nanoscale test bed for studies of photoinduced energy/charge transport and artificial photosynthesis and might find general use as elements for powering ultra-low-power electronics and diverse nanosystems [1-16].

\section{Outlook}

Building on our controlled design and synthesis of Si-NW PV elements with reproducible and carefully tunable PV properties, we have begun to explore increasing complex and novel NW tandem cell and quantum well structures in order to take rational steps toward enhancement of PV efficiency with builtin light-trapping structures. For example, precise control over radial dimensions allows us to insert, in principle, multiple quantum well (MQW) structures into the $i$-region of radial NWs (Fig. 5A). Quantum well solar cells (QWSCs) have been proposed as an option for increasing energy conversion efficiency above the Shockley-Queisser homojunction solar cell limit. The principle of operation centers around quantum wells, which act as efficient absorbers of photons, thereby raising the short-circuit current and energy conversion efficiency attainable. The open-circuit voltage of the NW QWSC can be designed to be dominated by the wider bandgap barrier material, while the short-circuit current can be determined by the width and depth of the quantum well (Fig. 5B). Initial efforts have led to the first synthesis of defect-free InGaN/GaN MQW structures [22,59] (Fig. 5C), and thus we believe that NWs are well
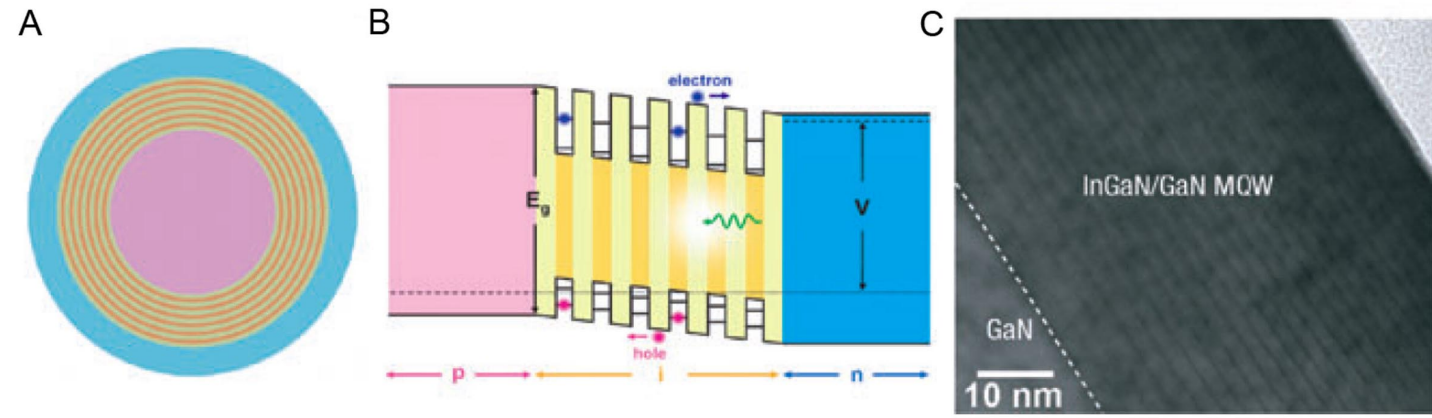

Fig. 5 New NW structures to improve energy conversion efficiency. A, Schematic of MQW NW solar cell. Pink and blue regions denote $p$ - core and $n$ - shell, respectively; yellow and orange regions are barriers and quantum wells in the $i$-shell, respectively. B, Band structure and carrier transport schematics of MQW NW solar cell. C, Brightfield TEM image of a $26 \mathrm{MQW}$ NW cross-section. The dashed line indicates the heterointerface between the core and shell. 
suited to implementing embedded MQW structures using materials which ordinarily could not be easily implemented on planar devices owing to issues of large lattice mismatch.

More generally, we believe that these and future fundamental studies at the single nanostructure level will be able to illuminate the key intrinsic properties, which if addressed could yield breakthroughs in efficiency, such that nano-enabled PVs might truly lead to a new generation of solar cells capable of providing economical power on a large scale.

\section{NANOWIRE CELLULAR PROBES}

Recording electrical signals from cells and tissue is central to areas ranging from the fundamental biophysical studies of function in, for example, the heart and brain, through medical monitoring and intervention. Over the past several decades, studies of electroactive cells and tissue have been carried out by using a variety of recording techniques [60-71], including glass micropipette intracellular and patchclamp electrodes [60,61], voltage-sensitive dyes [62], multielectrode arrays (MEAs) [63-65], and planar FETs [66-69]. The latter two recording techniques use well-developed microfabrication methods to allow for direct multiplexed detection on a scale not possible with micropipette technology, although the MEAs and FETs exhibit limited signal-to-noise ratios ( $/ \mathrm{N})$ and detection areas that make cellular and subcellular recording difficult [70,71]. A new class of molecular-scale electronic interfaces can be formed with cells and tissue using semiconductor NWs as functional elements.

\section{Brain slice recording}

Revealing the functional connectivity in natural neuronal networks is central to understanding circuits in the brain. We show that Si-NW FET arrays fabricated on transparent substrates via a bottom-up approach can be reliably interfaced to acute brain slices [23]. The structure of the array can be flexibly designed to address a wide range of experimental questions, while the transparent device chips enable imaging of individual cell bodies at both the upper and lower surfaces of the slices, and identification of areas of healthy pyramidal cells at the outmost tissue surface. Simultaneous, NW FET and patchclamp studies enabled unambiguous identification of action potential signals, with additional features detected at earlier times by the nanodevices. NW FET recording at different positions in the absence and presence of synaptic and ion-channel blockers enabled assignment of these features to presynaptic firing and postsynaptic depolarization from regions either close to somata or abundant in dendritic projections. In all cases, the NW FET signal amplitudes were from 0.3 to $3 \mathrm{mV}$. In contrast to conventional MEA measurements, the small active surface of the NW FET devices, $\sim 0.06 \mu \mathrm{m}^{2}$, provides highly localized multiplexed measurements of neuronal activities with demonstrated sub-millisecond temporal resolution and, significantly, better than $30 \mu \mathrm{m}$ spatial resolution. In addition, multiplexed mapping with 2D NW FET arrays revealed spatially heterogeneous functional connectivity in the olfactory cortex with a resolution surpassing substantially previous electrical recording techniques (Fig. 6) [23]. Our demonstration of simultaneous high temporal and spatial resolution recording, as well as mapping of functional connectivity, suggest that NW FETs can become a powerful platform for studying neural circuits in the brain.

In particular, our results demonstrate that the NW FET arrays detect local activity of the pyramidal cell layer and lateral olfactory tract (LOT) on at least the $10 \mu \mathrm{m}$ scale (Fig. 6), and thus can be used to understand the functional connectivity of this region [23]. The scale of local detection contrasts interdependent extracellular signals recorded from neighboring regions on even larger $(\sim 100 \mu \mathrm{m})$ length scales using MEAs. While it will be important to further describe the spatial and temporal limits of our NW FET arrays for acute brain slice recording, we believe that these results show clearly the potential of this tool for addressing critical biological problems. For example, the non-topographic fashion of fiber projection from the olfactory bulb to the cortex makes fine anatomic analysis of connections across functional regions difficult [72,73], and while retrograde labeling and transneuronal genetic tracing can 
A
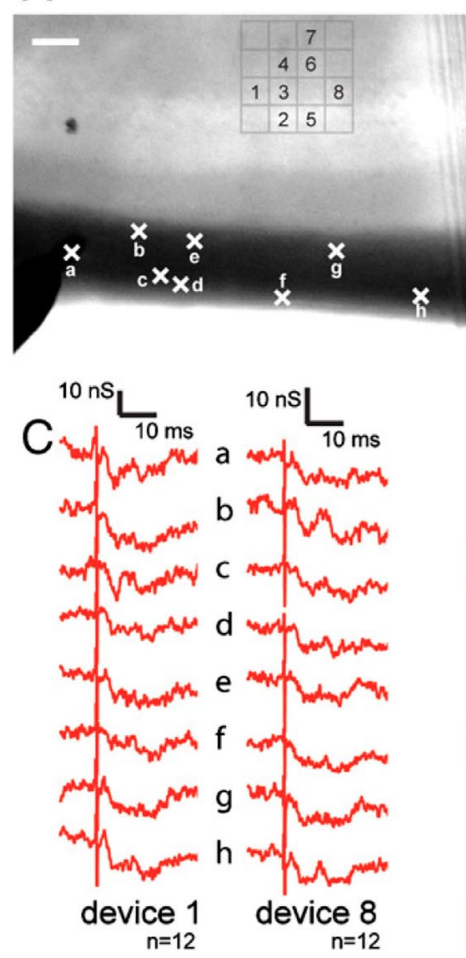

E

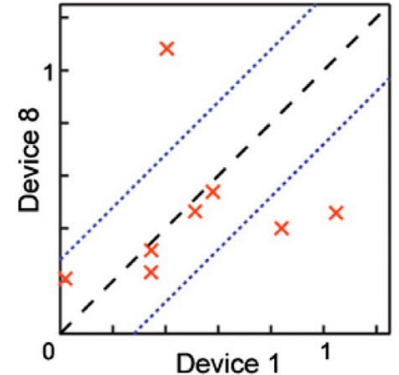

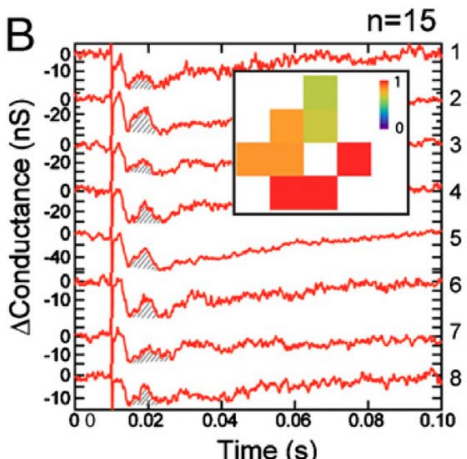
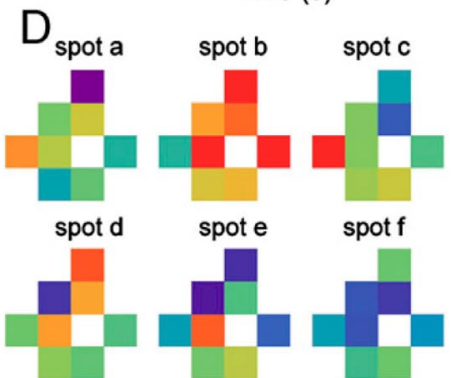

spote $\quad$ spotf

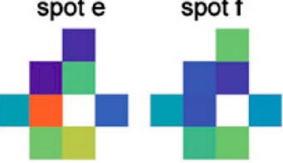

spot h
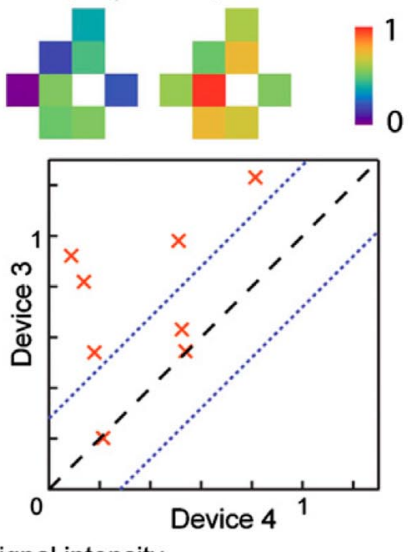

Fig. 6 2D mapping of heterogeneous activities in the pyramidal cell layer. A, Optical image of an acute slice over a $4 \times 4$ NW FET array. Signals were recorded simultaneously from the 8 devices indicated on the image. Crosses along the LOT fiber region of the slice mark the stimulation spots $\boldsymbol{a}$ through $\boldsymbol{h}$. The stimulator insertion depth was not controlled precisely in these experiments. Scale bar represents $100 \mu \mathrm{m}$. B, Signals recorded for devices 1-8 when stimulated with a $200 \mu \mathrm{s} 400 \mu \mathrm{A}$ pulse. Data are averaged from 15 recordings. The shaded area in each trace corresponds to the $p$-spike and was used to obtain normalized intensity (see Methods). Inset: normalized map of the signal intensity from the 8 devices. C, Representative signals recorded from devices 1 and 8 when stimulating at spots $\boldsymbol{a}$ through $\boldsymbol{h}$, with $200 \mu \mathrm{s}, 100 \mu \mathrm{A}$ pulse. Data are averaged from 12 recordings. D, Maps of the relative signal intensity or activity for devices $1-8$. E, Correlation between devices 1 and 8 (upper plot) and devices 3 and 4 (lower plot) for the different stimulation positions. The dashed black line marks signals that are correlated. The dotted blue lines mark the uncertainty owing to device signal fluctuations determined from correlation analysis. 
potentially provide necessary anatomical information, the functional connectivity of the olfactory cortex remains largely unknown [74]. Furthermore, the plasticity of the olfactory system suggests that the network is highly dynamic. Thus, highly localized direct recording of ensembles of neurons in the context of spatially resolved stimulation could serve as a powerful tool to visualize the dynamic, functional connection map and provide information necessary to understand the circuits and plasticity in this and other neural systems.

In addition, the use of surface-passivated NW FETs circumvents the common challenge confronted by implanted microelectrodes, where post-implant increases of impedance caused by, for example, absorption of proteins, leads to degraded signal quality and higher noise level [75]. This makes NW FETs very promising for high signal yield, chronic in vivo recordings, especially considering the facts that (1) small device feature size allows multiplexed detectors being integrated onto ultra-small probes for minimum damage to the tissue, (2) bottom-up fabrication makes it possible to choose biocompatible materials as substrates to reduce mechanical mismatch and to minimize reactive tissue response [76,77], and (3) the nanoscale morphology could promote better attachment of active neurons, leading to better signal quality than planar designs. Therefore, NW FETs could also bring exciting new opportunities to interfacing living tissue and electronics.

\section{Intracellular recording}

Patch-clamp micropipettes [78,79] and carbon microelectrodes [80-82] are examples of single-terminal probes used for intracellular electrical and electrochemical recording, respectively. The single electrical connection facilitates design and mechanical insertion into cells, but these probes also have limitations in terms of ultimate size and/or exposure of cells to internal pipette solution [79]. On the other hand, FETs could function in the $5 \mathrm{~nm}$ size regime [83], although the requirement of two electrical connections [83] makes minimally invasive insertion into a cell a substantial challenge. To develop a twoterminal semiconductor device that has both nanometer dimensions and capability for facile insertion into single cells, we have designed and synthesized single-crystalline dopant-modulated Si-NW structures with acute probe tip angles (Fig. 7A). Conceptually, our design is based on recent observation that variation of reactant pressure during NW growth could introduce reproducible $120^{\circ}$ kinks in Si-NWs [17] and the fact that selective incorporation of two or three cis-linked kinked units will yield probe tip angles of $60^{\circ}$ or $0^{\circ}$, respectively (Fig. 7A, top and middle, Fig. 7B). Because two trans-linked units (Fig. 7A, bottom) would yield an unusable probe tip, the selective synthesis of cis-linked units is central to our design [24].

To test these NW FET probes in a 3D geometry capable of making intracellular interfaces, we designed a straightforward fabrication approach in which (i) source and drain connections to the FET probes were made above a sacrificial layer and (ii) the interfacial stress between materials [84] was used to bend the probe upwards as illustrated in Fig. 7C. A representative SEM image of one free-standing 3D device (Fig. 7C) demonstrates that the $60^{\circ}$ kinked probe is intact after fabrication with the two NW arm terminals sandwiched between SU-8 polymer and metal contacts. This suggests that single-crystalline NW probes are robust, and indeed, we have achieved $\geq 90 \%$ yields with $\sim 30$ nanoprobe devices per chip. In addition, the probe height and angle (Fig. 7C) can be systematically tuned by changing the length and thickness of the free-standing part of the metal contact/SU-8 structure. Last, free-standing 3D FET devices have been stored in air for at least 8 months without substantial change in nanoprobe orientations and FET sensitivity.

Finally, we have investigated the formation of intracellular interfaces between our 3D NW FET probes and spontaneously firing electrogenic cells. Embryonic chicken cardiomyocytes were cultured on poly(dimethylsiloxane) PDMS substrates [85] and then positioned to place individual cells over phospholipid-modified vertical NW probes within a cell perfusion chamber. Representative conductance vs. time data recorded from a 3D FET probe initially in gentle contact with a spontaneously beating cardiomyocyte cell showed a sequence of distinct features (Fig. 7D). Initially, we observed regu- 
A

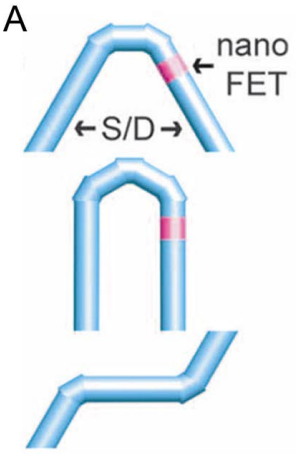

B

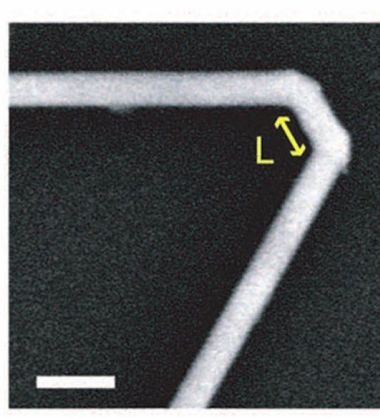

C

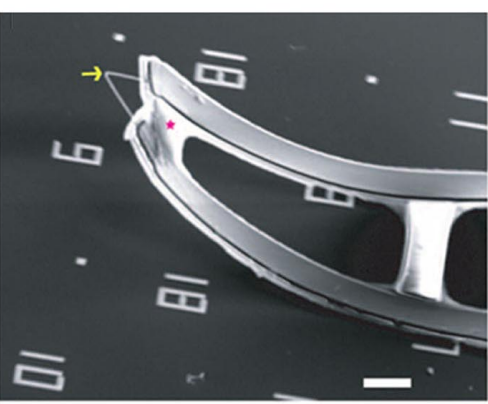

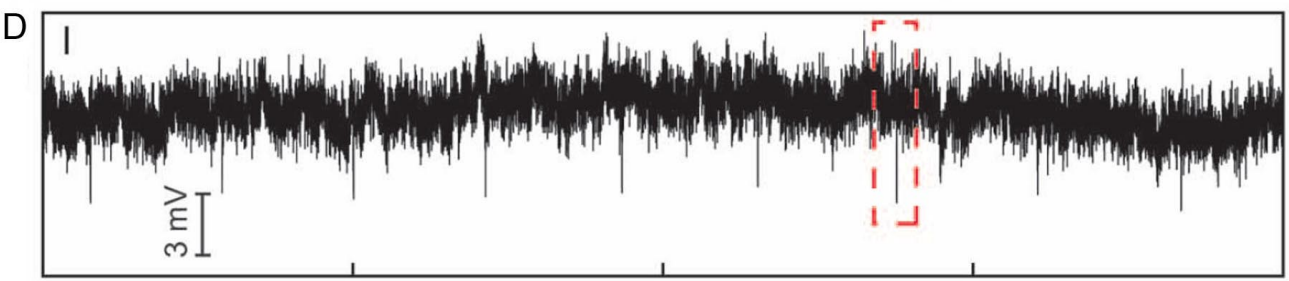
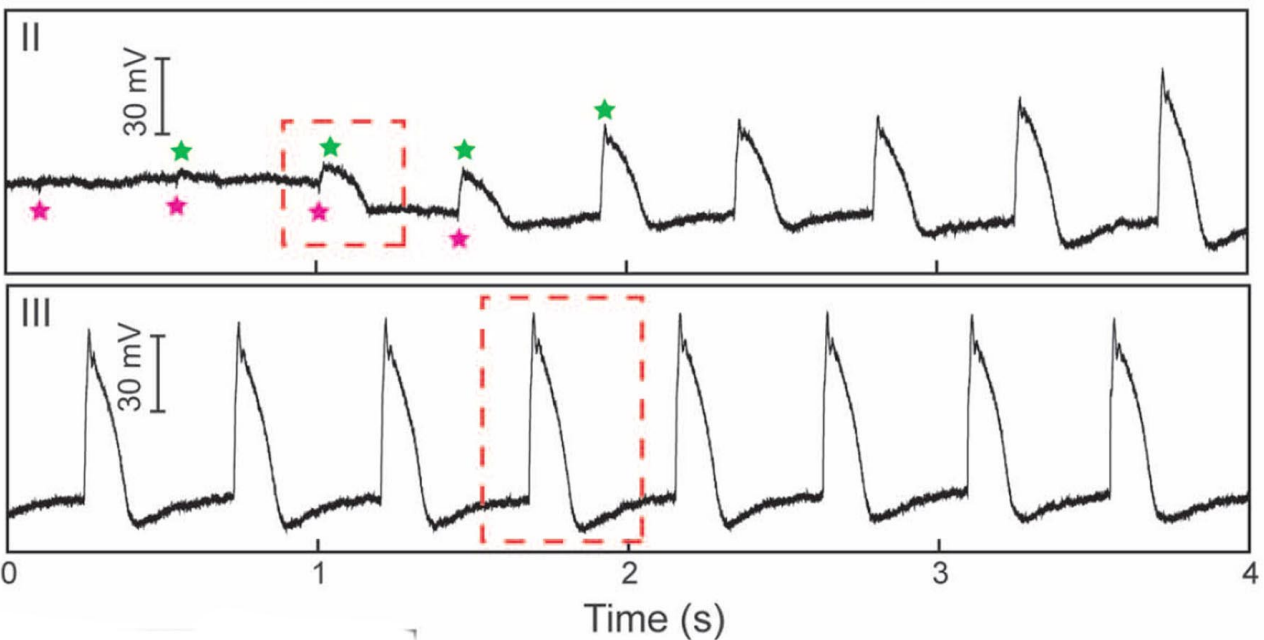

Fig. 7 Nanoscale intracellular FET probes. A, Schematics of $60^{\circ}$ (top) and $0^{\circ}$ (middle) multiply kinked NWs and cis (top) and trans (bottom) configurations in NW structures. The blue and pink regions designate the source/drain (S/D) and nanoscale FET channel, respectively. B, SEM image of a doubly kinked NW with a cis configuration. $L$ is the length of segment between two adjacent kinks. Scale bar, $200 \mathrm{~nm}$. C, SEM of an as-made device. The yellow arrow and pink star mark the nanoscale FET and SU-8, respectively. Scale bars, $5 \mu \mathrm{m}$. D, Electrical recording from beating cardiomyocytes: (i) extracellular recording, (ii) transition from extracellular to intracellular recordings during cellular entrance, and (iii) steady-state intracellular recording. Green and pink stars denote the peak positions of intra- and extracellular signal components, respectively.

larly spaced spikes with a frequency of ca. $2.3 \mathrm{~Hz}$ consistent with beating cardiomyocyte (Fig. 7D, I). The peaks have a potential change of $\sim 3-5 \mathrm{mV}, \mathrm{S} / \mathrm{N} \geq 2$, and a sub-millisecond width (Fig. 7D, I). The peak amplitude, shape, and width are similar to extracellular recordings made with NW devices on substrates, and, moreover, optical images recorded at the same time (Fig. 7D, I) are consistent with extracellular signals. These results highlight our ability to achieve extracellular recordings using a truly nanoscale and free-standing detector, and suggest the potential to interfacing with biological tissues such as brain slices [23] in 3D. 
Following a relatively brief ( $40 \mathrm{~s})$ period of extracellular signals, we observed several pronounced changes in recorded signals (Fig. 7D, II and III) without application of external force to the PDMS/cell support. Specifically, the initial extracellular signals gradually disappear (Fig. 7D, II, magenta stars) with a concomitant decrease in baseline potential and emergence of new peaks that have an opposite sign, much larger amplitude, and longer duration (Fig. 7D, II, green stars). These new peaks, which are coincident with cell beating, rapidly reach a steady state (Fig. 7D, III) with an average calibrated peak amplitude of $\sim 80 \mathrm{mV}$ and duration of $\sim 200 \mathrm{~ms}$. The amplitude, sign, and duration are close to those reported for whole-cell patch-clamp recordings from cardiomyocytes [86], and thus we conclude that these data represent a transition to steady-state intracellular recording with the 3D NW probe.

There are several other important characteristics of our new 3D NW FET bioprobes that can be gleaned from these experiments. First, the regular intracellular recording (e.g., Fig. 7D, III) from the cardiomyocytes over periods up to 10 min suggests a secure NW/cell membrane seal and a flexible recording capability using the phospholipids layers even with mechanical deformations associated with beating. Second, close inspection of the transitional recordings (Fig. 7D, II) reveals that the switching from extra- to intracellular signals is smooth and without appreciable change in frequency. This result shows that internalization of the NW probe tip is not invasive and has no perturbation on the electrogenic cell-firing patterns. We believe that this behavior is a result of small sizes of the kinked NW probes and their biomimetic phospholipid surfaces. Third, the disappearance of extracellular signals, after the intracellular ones become dominant, demonstrates that the extracellular exposed contacts and heavily doped Si-NW arms do not contribute significantly to the observed signals, and further indicates the secure NW/cell membrane seal. Moreover, these results confirm that electrical recording arises from the highly localized nanoscale FET segment near the probe tip, which (a) initially records only extracellular potential, (b) simultaneously records both extracellular and intracellular signals as the FET spans the cell membrane, and (c) records only intracellular signals when fully inside the cell. Last, the transitional data directly show that the extracellular spikes are aligned where intracellular sodium influx initiates (Fig. 7D, II) and are temporally separated from the cardiac contraction [86].

\section{Outlook}

We believe that the design and realization of NW cellular probes, in particular, the kinked NW FET probes, open up substantial opportunity as a new biomaterial. Significantly, our work demonstrates for the first time the capability of intracellular recording with a semiconductor device, and shows that these phospholipid-modified nanoprobes enable such intracellular measurements with high-resolution and minimal mechanical perturbation of cells. Additional work remains in order to develop this new biomaterial to be a routine tool like the patch-clamp micropipette, although we believe that there are already clear advantages; that is, recording is relatively simple without the need for resistance or capacitance compensation, the kinked NW probes are mechanically and chemically (i.e., no solution exchange with pipette) less invasive, and very high spatial/temporal resolution can be achieved. The capability to detect selectively different biological and chemical species and multiplexed measurements within a single cell is also unique to these active devices. More generally, the nanometer size, biomimetic surface coating, and flexible 3D geometry suggest future biomedical applications where the distinctions between electronic devices and living cells are blurred.

\section{REFERENCES}

1. J. T. Hu, T. W. Odom, C. M. Lieber. Acc. Chem. Res. 32, 435 (1999).

2. C. M. Lieber. MRS Bull. 28, 486 (2003).

3. Y. Cui, X. Duan, Y. Huang, C. M. Lieber. In Nanowires and Nanobelts: Materials, Properties and Devices, Z. L. Wang (Ed.), Kluwer Academic (2003). 
4. Y. Huang, C. M. Lieber. Pure Appl. Chem. 76, 2051 (2004).

5. L. J. Lauhon, M. S. Gudiksen, C. M. Lieber. Philos. Trans. R. Soc. London, Ser. A 362, 1247 (2004).

6. L. Samuelson. Mater. Today 6, 22 (2003).

7. Y. N. Xia, P. D. Yang, Y. G. Sun, Y. Y. Wu, B. Mayers, B. Gates, Y. D. Yin, F. Kim, Y. Q. Yan. Adv. Mater. 15, 353 (2003).

8. Z. L. Wang. Mater. Today 7, 26 (2004).

9. M. Bruchez Jr., M. Moronne, P. Gin, S. Weiss, A. P. Alivisatos. Science 281, 2013 (1998).

10. C. B. Murray, C. R. Kagan, M. G. Bawendi. Annu. Rev. Mater. Sci. 30, 545 (2000).

11. X. Wang, J. Zhuang, Q. Peng, Y. D. Li. Nature 437, 121 (2005).

12. T. W. Odom, J. L. Huang, P. Kim, C. M. Lieber. J. Phys. Chem. B 104, 2794 (2000).

13. M. Ouyang, J. L. Huang, C. M. Lieber. Acc. Chem. Res. 35, 1018 (2002).

14. M. Ouyang, J. L. Huang, C. M. Lieber. Annu. Rev. Phys. Chem. 53, 201 (2002).

15. J. Liu, S. S. Fan, H. J. Dai. MRS Bull. 29, 244 (2004).

16. P. L. McEuen, J. Y. Park. MRS Bull. 29, 272 (2004).

17. B. Z. Tian, P. Xie, T. J. Kempa, D. C. Bell, C. M. Lieber. Nat. Nanotechnol. 4, 824 (2009).

18. X. C. Jiang, B. Z. Tian, J. Xiang, F. Qian, G. F. Zheng, H. T. Wang, C. M. Lieber. Proc. Natl. Acad. Sci. USA 108, 12212 (2011).

19. B. Z. Tian, T. J. Kempa, C. M. Lieber. Chem. Soc. Rev. 38, 16 (2009).

20. B. Z. Tian, X. L. Zheng, T. J. Kempa, Y. Fang, N. F. Yu, G. H. Yu, J. L. Huang, C. M. Lieber. Nature 449, 885 (2007).

21. T. J. Kempa, B. Z. Tian, D. R. Kim, J. S. Hu, X. L. Zheng, C. M. Lieber. Nano Lett. 8, 3456 (2008).

22. Y. J. Dong, B. Z. Tian, T. J. Kempa, C. M. Lieber. Nano Lett. 9, 2183 (2009).

23. Q. Qing, S. K. Pal, B. Z. Tian, X. J. Duan, B. P. Timko, T. Cohen-Karni, V. N. Murthy, C. M. Lieber. Proc. Natl. Acad. Sci. USA 107, 1882 (2010).

24. B. Z. Tian, T. Cohen-Karni, Q. Qing, X. J. Duan, P. Xie, C. M. Lieber. Science 329, 830 (2010).

25. R. S. Wagner, W. C. Ellis. Appl. Phys. Lett. 4, 89 (1964).

26. A. M. Morales, C. M. Lieber. Science 279, 208 (1998).

27. J. L. Liu, S. J. Cai, G. L. Jin, S. G. Thomas, K. L. Wang. J. Cryst. Growth 200, 106 (1999).

28. K. Haraguchi, T. Katsuyama, K. Hiruma, K. Ogawa. Appl. Phys. Lett. 60, 745 (1992).

29. X. F. Duan, C. M. Lieber. Adv. Mater. 12, 298 (2000).

30. M. S. Gudiksen, L. J. Lauhon, J. Wang, D. C. Smith, C. M. Lieber. Nature 415, 617 (2002).

31. L. J. Lauhon, M. S. Gudiksen, D. L. Wang, C. M. Lieber. Nature 420, 57 (2002).

32. F. Qian, Y. Li, S. Gradečak, D. L. Wang, C. J. Barrelet, C. M. Lieber. Nano Lett. 4, 1975 (2004).

33. C. Yang, Z. H. Zhong, C. M. Lieber. Science 310, 1304 (2005).

34. R. E. Algra, M. A. Verheijen, M. T. Borgström, L.-F. Feiner, G. Immink, W. J. P. Van Enckevort, E. Vlieg, E. P. A. M. Bakkers. Nature 456, 369 (2008).

35. P. Caroff, K. A. Dick, J. Johansson, M. E. Messing, K. Deppert, L. Samuelson. Nat. Nanotechnol. 4, 50 (2009).

36. F. M. Davidson, D. C. Lee, D. D. Fanfair, B. A. Korgel. J. Phys. Chem. C 111, 2929 (2007).

37. F. M. Ross, J. Tersoff, M. C. Reuter. Phys. Rev. Lett. 95, 146104-1 (2005).

38. P. X. Gao, Y. Ding, W. J. Mai, W. L. Hughes, C. S. Lao, Z. L. Wang. Science 309, 1700 (2005).

39. W. Lu, C. M. Lieber. Nat. Mater. 6, 841 (2007).

40. F. Patolsky, B. P. Timko, G. Zheng, C. M. Lieber. MRS Bull. 32, 142 (2007).

41. O. M. Yaghi, M. O’Keeffe, N. W. Ockwig, H. K. Chae, M. Eddaoudi, J. Kim. Nature 423, 705 (2003).

42. N. S. Lewis. Science 315, 798 (2007). 
43. Basic Research Needs for Solar Energy Utilization, Report of the Basic Energy Sciences Workshop on Solar Energy Utilization. April 18-21, 2005, U.S. Department of Energy, Washington, DC (2005). <www.er.doe.gov/bes/reports/abstracts.html\#SEU>

44. Form EIA-63B, Annual Photovoltaic Module/Cell Manufacturers Survey, Energy Information Administration, United States (2006).

45. D. Ginley, M. A. Green, R. Collins. MRS Bull. 33, 355 (2008).

46. S. E. Shaheen, D. S. Ginley, G. E. Jabbour. MRS Bull. 30, 10 (2005).

47. M. Grätzel. MRS Bull. 30, 23 (2005).

48. C. J. Brabec, J. A. Hauch, P. Schilinsky, C. Waldauf. MRS Bull. 30, 50 (2005).

49. F. Dimroth, S. Kurtz. MRS Bull. 32, 230 (2007).

50. R. R. King, D. C. Law, K. M. Edmondson, C. M. Fetzer, G. S. Kinsey, H. Yoon, R. A. Sherif, N. H. Karam. Appl. Phys. Lett. 90, 183516 (2007).

51. A. Luque, A. Marti, A. J. Nozik. MRS Bull. 32, 236 (2007).

52. Y. Li, F. Qian, J. Xiang, C. M. Lieber. Mater. Today 9, 18 (2006).

53. M. Grätzel. Nature 414, 338 (2001).

54. W. U. Huynh, J. J. Dittmer, A. P. Alivisatos. Science 295, 2425 (2002).

55. M. Law, L. E. Greene, J. C. Johnson, R. Saykally, P. Yang. Nat. Mater. 4, 455 (2005).

56. J. B. Baxter, E. S. Aydil. Appl. Phys. Lett. 86, 053114 (2005).

57. B. M. Kayes, H. A. Atwater, N. S. Lewis. J. Appl. Phys. 97, 114302 (2005).

58. S. M. Sze. Physics of Semiconductor Devices, $2^{\text {nd }}$ ed., John Wiley (2004).

59. F. Qian, Y. Li, S. Gradečak, H.-G. Park, Y. Dong, Y. Ding, Z. L. Wang, C. M. Lieber. Nat. Mater. 7, 701 (2008).

60. B. Hille. Ion Channels of Excitable Membranes, Sinauer, Sunderland, MA (2001).

61. J. T. Davie, M. H. P. Kole, J. J. Letzkus, E. A. Rancz, N. Spruston, G. J. Stuart, M. Häusser. Nat. Protocols 1, 1235 (2006).

62. A. G. Kléber, Y. Rudy. Physiol. Rev. 84, 431 (2003).

63. T. Meyer, K. H. Boven, E. Gunther, M. Fejtl. Drug Safety 27, 763 (2004).

64. J. Erickson, A. Tooker, Y.-C. Tai, J. Pine. J. Neurosci. Methods 175, 1 (2008).

65. J. K. Y. Law, C. K. Yeung, B. Hofman, S. Ingebrandt, J. A. Rudd, A. Offenhäusser, M. Chan. Physiol. Meas. 30, 155 (2009).

66. S. Ingebrandt, C.-K. Yeung, M. Krause, A. Offenhausser. Biosens. Bioelectron. 16, 565 (2001).

67. C.-K. Yeung, S. Ingebrandt, M. Krause, A. Offenhausser, W. Knoll. J. Pharmacol. Toxicol. Methods 45, 207 (2001).

68. A. Cohen, J. Shappir, S. Yitzchiak, M. E. Spira. Biosens. Bioelectron. 22, 656 (2006).

69. A. Cohen, J. Shappir, S. Yitzchaik, M. E. Spira. Biosens. Bioelectron. 23, 811 (2008).

70. Z.-L. Lu, A. Pereveraev, H.-L. Liu, M. Weiergräber, M. Henry, A. Krieger, N. Smyth, J. Hescheler, T. Schneider. J. Electrocardiol. 14, 11 (2004).

71. M. Reppel, F. Pillekamp, Z. J. Lu, M. Halbach, K. Brockmeier, B. K. Fleischmann, J. Hescheler. J. Electrocardiol. 37, 104 (2004).

72. M. Devor. J. Comp. Neurol. 166, 31 (1976).

73. J. W. Scott, R. L. McBride, S. P. Schneider. J. Comp. Neurol. 194, 519 (1980).

74. A. Martinez-Marcos. Prog. Neurobiol. 87, 21 (2009).

75. A. Mercanzini, P. Colin, J. C. Bensadoun, A. Bertsch, P. Renaud. IEEE Trans. Biomed. Eng. 56, 1909 (2009).

76. D. H. Kim, J. H. Ahn, W. M. Choi, H. S. Kim, T. H. Kim, J. Z. Song, Y. G. Y. Huang, Z. J. Liu, C. Lu, J. A. Rogers. Science 320, 507 (2008).

77. D. H. Kim, Y. S. Kim, J. Amsden, B. Panilaitis, D. L. Kaplan, F. G. Omenetto, M. R. Zakin, J. A. Rogers. Appl. Phys. Lett. 95, 133701 (2009).

78. B. Sakmann, E. Neher. Ann. Rev. Physiol. 46, 455 (1984). 
79. A. Molleman. Patch Clamping: An Introductory Guide to Patch Clamp Electrophysiology, John Wiley, New York (2002).

80. R. M. Wightman. Science 311, 1570 (2006).

81. A. G. Ewing, T. G. Strein, Y. Y. Lau. Acc. Chem. Res. 25, 440 (1992).

82. M. G. Schrlau, N. J. Dun, H. H. Bau. ACS Nano 3, 563 (2009).

83. Z. H. Zhong, Y. Fang, W. Lu, C. M. Lieber. Nano Lett. 5, 1143 (2005).

84. T. G. Leong, C. L. Randall, B. R. Benson, N. Bassik, G. M. Stern, D. H. Gracias. Proc. Natl. Acad. Sci. USA 106, 703 (2009).

85. T. Cohen-Karni, B. P. Timko, L. E. Weiss, C. M. Lieber. Proc. Natl. Acad. Sci. USA 106, 7309 (2009).

86. D. P. Zipes, J. Jalife. Cardiac Electrophysiology: From Cell to Bedside, $5^{\text {th }}$ ed., Saunders, Philadelphia (2009). 